\title{
Determinacion del efecto hipocolesterolemico y antiaterosclerotico de extracto acuoso de fruto Solanum Melongena "berenjena" en Rattus norvegicus var. Wistar con hiperlipemia experimental.
}

Determination of the hypocolesterolemic and anti-stereocherotic effect of aquatic fruit extract Solanum melongena "berenjena" in Rattus norvegicus var. Wistar with experimental hyperlipemia.

Luis Enrique Moncada Bernedo y Henry Diaz-Murillo

Universidad Nacional de San Agustín de Arequipa, Arequipa Perú.

\section{INFORMACIÓN}

\section{Historia del Artículo}

Recepción: 09/08/2019

Revisión: 03/10/2019

Aceptación: 01/11/2019

\section{Palabras Clave}

Solanum melongena "berenjena", Rattus norvegicus. var. Wistar, hipercolesterolémico.

\section{Key Words}

Solanum melongena "berenjena", Rattus norvegicus. var. Wistar. Hypercholesterolemic.

\section{DOI}

https://doi.org/10.35286/veritas. v21i1.263

\begin{abstract}
RESUMEN
Se evaluó la actividad hipolipemiante del extracto acuoso de fruto de Solanum melongena "berenjena" en Rattus norvegicus variedad Wistar con hiperlipidemia experimental. Se llevó a cabo en el Laboratorio de Fisiología Animal de la Univerisdad Nacional de San Agustín y Bioterio de la Universidad Católica Santa María de 1 Junio al 31 Setiembre 2015. Se utilizaron 24 Rattus norvegicus variedad Wistar distribuidas en cuatro grupos de 6 ratas cada uno: un tratamiento control hipercolesterolémico y tres experimentales tratamiento de Solanum melongena a dosis de $0.5 \mathrm{~g} / \mathrm{kg} / \mathrm{día}, 1 \mathrm{~g} / \mathrm{kg} /$ día y lovastatina a una dosis de $3 \mathrm{mg} / \mathrm{kg}$. Se utilizó el método de Wiener en la determinación de Colesterol Total, Triglicéridos, HDL-colesterol y LDL-colesterol. La hiperlipidemia se logró con una mezcla de $50 \mathrm{~g}$ de cerebro de res hervido con $50 \mathrm{~g}$ de conejina más $3 \mathrm{ml}$ de manteca de cerdo por rata. Se observó disminución significativa $(\mathrm{p}<0.01)$ en los niveles de colesterol, triglicéridos e índice aterogénico y aumento significativo en los niveles de HDL-C, en comparación al grupo control. Los resultados nos muestran que la administración de 0.5 y $1 \mathrm{~g} / \mathrm{kg} /$ día de extracto acuoso de Solanum melongena "berenjena" disminuyen los niveles de colesterol total y triglicéridos en Rattus norvegicus variedad Wistar con hiperlipidemia experimental. Ademas no tiene efecto hepatotoxico y reduce las placas ateromatosas en aorta.
\end{abstract}

\begin{abstract}
The lipid-lowering activity of the aqueous extract of Solanum melongena "berenjena" fruit in Rattus norvegicus Wistar variety with experimental hyperlipidemia was evaluated. It was carried out in the Animal Physiology Laboratory of the National University of San Agustín and Bioterio of the Universidad Católica de Santa María from June 1 to September 31, 2015. 24 Rattus norvegicus Wistar variety distributed in four groups of 6 rats each were used: a hypercholesterolemic control treatment and three experimental Solanum melongena treatment at a dose of $0.5 \mathrm{~g} / \mathrm{kg} /$ day, $1 \mathrm{~g} / \mathrm{kg} /$ day and lovastatin at a dose of $3 \mathrm{mg} / \mathrm{kg}$ The Wiener method was used in the determination of Total Cholesterol, Triglycerides, HDL-cholesterol and LDLcholesterol. Hyperlipidemia was achieved with a mixture of $50 \mathrm{~g}$ of boiled beef brain with $50 \mathrm{~g}$ of rabbits plus $3 \mathrm{ml}$ of lard per rat. A significant decrease $(\mathrm{p}<0.01)$ was observed in cholesterol, triglyceride and atherogenic index levels and a significant increase in HDL-C levels, compared to the control group. The results show us that the administration of 0.5 and $1 \mathrm{~g} / \mathrm{kg} /$ day of aqueous extract of Solanum melongena "berenjena" decreases the levels of total cholesterol and triglycerides in Rattus norvegicus Wistar variety with experimental hyperlipidemia. It also has no hepatotoxic effect and reduces atheromatous plaques in the aorta.
\end{abstract}

\section{INTRODUCCIÓN}

La hiperlipidemia es una condición producida por elevación de la concentración de constituyentes lipídicos de la sangre denotando, generalmente, exceso de colesterol, trigliceridos o de ambos. Este desequilibrio ocasiona preocupación clínica, ya que constituye un factor de riesgo en enfermedades cardiovasculares, acelerando el desarrollo de aterosclerosis, con sus secuelas de trombosis e infarto (Rubin 2010).
Las enfermedades cardiovasculares, constituyen la primera causa de muerte de la población adulta en países latinoamericanos, llegando a ser la causa de muerte principal en algunos de ellos.

El alto costo que representa la adquisición de los fármacos utilizados para el control de la hiperlipidemia, hace que estos productos sean muchas veces inaccesibles a gran parte de la población que padece dicha enfermedad.

Hoy en día, la base del tratamiento de la hiperlipidemia se inicia siempre con terapias no farmacológicas como el manejo de la dieta y otros factores de riesgo (tabaquismo, alcohol, etc) en caso de no obtener resultados favorables, se emplean terapias farmacológicas haciendo uso de resinas de intercambio iónico (colestipol, colestiramina), fibratos (gemfibrozil, clofibrato, bezafibrato, etofibrato); inhibidores de la hidroximetilglutarilCoA reductasa (lovastatina, 
simvastatina, provastatina, fluvastatina); vitaminas (niacina, ácido nicotínico); ácidos grasos omega 3 , etc (Goodman 2002).

Se ha reportado que Solanum melongena "berenjena" en sus principios activos tiene sustancias (acido esteárico, ácido clorogénico, ácido linoleico, fitosterol, licopeno, magnesio, cromo, cobre, calcio, niacina, fibra, pectina, trigonelina, etc.) con actividad hipolipemiante. hipocolesterolemiante, hipotrigliceridemiante (Duke 1992).

A fin de contribuir con la busqueda de nuevos agentes hipolipemiantes, es que proponemos la investigación del efecto del extracto de Solanum melongena "berenjena" en el perfil lipídico en Rattus norvegicus variedad Wistar.

\section{MATERIAL Y MÉTODOS}

\section{Lugar}

Laboratorio de Fisiología Animal de la Universidad Nacional de San Agustín y Bioterio de la Universidad Católica Santa María de Junio a Setiembre del 2015.

\section{Unidades de estudio}

Se emplearon 24 ratas de la especie Rattus norvegicus variedad Wistar de 4 a 5 meses edad, con pesos que fluctúan entre 300 a $350 \mathrm{~g}$ distribuidos en 4 grupos de 6 ratas cada uno. La hiperlipidemia se indujo con una mezcla de $50 \mathrm{~g}$ de cerebro de res hervido con $50 \mathrm{~g}$ de conejina más $3 \mathrm{ml}$ de manteca de cerdo por rata.

\begin{tabular}{cc}
\hline GRUPOS & UNIDADES EXPERIMENTALES \\
\hline CONTROL & 6 \\
Solanum melongena $0.5 \mathrm{~g} / \mathrm{Kg} / \mathrm{dia}$ & 6 \\
Solanum melongena $1 \mathrm{~g} / \mathrm{Kg} / \mathrm{dia}$ & 6 \\
Lovastatina $3 \mathrm{mg} / \mathrm{Kg} / \mathrm{dia}$ & 6 \\
TOTAL & 24 \\
\hline
\end{tabular}

Para la preparación del extracto acuoso de Solanum melongena se utilizaron frutos $(50 \mathrm{~g}$.), con $100 \mathrm{ml}$. de agua destilada despues se licuaron por 3 minutos. El licuado se filtró con una gasa posteriormente el filtrado se guardo en un frasco de vidrio oscuro y en refrigeración para ser usado durante un plazo máximo de tres días.

Las preparaciones obtenidas fueron administradas por vía orogástrica usando una jeringa metálica especial en las dosis siguientes:

- $0.5 \mathrm{~g} / \mathrm{Kg} / \mathrm{dia}$.

- $1 \mathrm{~g} / \mathrm{Kg} /$ dia.

Para la lovastatina se diluye una pastilla de $300 \mathrm{mg}$. en $50 \mathrm{ml}$. de agua destilada y se guardó en un frasco de vidrio oscuro, en refrigeración; luego se realiza el cálculo respectivo, por peso del animal y de esta manera se extrae la cantidad necesaria.

\section{Obtención de la muestra de sangre}

La rata se colocó en una trampa de madera de donde sólo debe sobresalir la cola. La muestra de sangre se obtendrá directamente de la punta de la cola de la rata, se desinfecta previamente con alcohol y se realiza un corte de la cola con una hoja de bisturí estéril, la sangre es colectada en capilares heparinizados.

\section{Determinación del perfil lipidico \\ Determinación de colesterol método chod-pap}

El colesterol se determina por acción de las enzimas colesterol éster hidrolasa y colesterol oxidasa. La primera libera el colesterol de los ésteres de colesterol, y la segunda oxida el colesterol libre produciéndose peróxido de hidrógeno, el cual en presencia de la enzima peroxidasa reacciona con el sistema cromogénico dando origen a un compuesto coloreado que absorbe a $505 \mathrm{~nm}$.

\section{Reactivos}

- Stándar de colesterol $200 \mathrm{mg} / \mathrm{dl}$. en solución acuosa estabilizada.

- Enzimas: Suspensión conteniendo colesterol éster hidrolasa, colesterol oxidasa, peroxidasa.

- Reactivo de 4-aminoantipirina (0.4 mM), Ac. p-hidroxibenzoico (10 $\mathrm{mM})$ y Buffer fosfato $(90 \mathrm{mM})$.

\section{Técnica}

Utilizar de preferencia plasma libre de hemólisis, de muestras de animales en ayunas. En tubos de ensayo limpios $\mathrm{y}$ añadir.

\begin{tabular}{cccc}
\hline & BLANCO & ESTANDAR & MUESTRA \\
\hline MUESTRA (ul) & $-\cdots--$ & $-\cdots--$ & $10 \mathrm{ul}$. \\
ESTANDAR (ul) & ---- & $10 \mathrm{ul}$. & $-\cdots$ \\
REACTIVO (ml) & $1 \mathrm{ml}$. & $1 \mathrm{ml}$. & $1 \mathrm{ml}$. \\
\hline
\end{tabular}

Mezclar e incubar por $10 \mathrm{~min}$. a $37^{\circ} \mathrm{C}$ (Baño María), ó 20 min. a temperatura ambiente $\left(20\right.$ a $\left.25^{\circ} \mathrm{C}\right)$.

Leer las absorbancias a $505 \mathrm{~nm}$. Llevando a cero el espectrofotómetro con el reactivo de trabajo (blanco)

El color resultante es estable por al menos $30 \mathrm{~min}$.

\section{Cálculos}

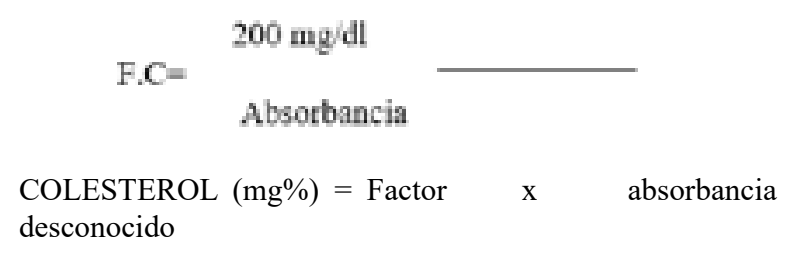




\section{Determinación de triglicéridos -método wiener}

Los triglicéridos son hidrolizados por una lipasa específica liberando ácidos grasos y glicerol. El glicerol es fosforilado por la enzima gliceroquinasa y posteriormente, el glicerol-l-fosfato es oxidado a dihidroxiacetona fosfato por la enzima glicerol fosfato oxidasa, generándose peróxido de hidrógeno. Posteriormente en una reacción del tipo Trinder, el peróxido de hidrógeno reacciona con 4-aminoantipirina y el ácido 3,5-dicloro-2-hidroxi-bencensulfónico para producir por medio de la enzima peroxidasa un compuesto coloreado en cantidad proporcional a la concentración de triglicéridos presente en la muestra, midiéndose la absorbancia a $520 \mathrm{~nm}$.

\section{Reactivos}

Stándar de Glicerol en solución estabilizada equivalente a $200 \mathrm{mg} / \mathrm{dl}$. de triglicéridos.

Enzimas: Lipasa (microbial), glicerokinasa, glicerol-3fosfato oxidasa y peroxidasa.

Reactivo de 4-aminoantipirina (1 mM), Ac. 3,5-dicloro2-hidroxi-bencensulfónico (1.5mM), Mg+2 (5 mM) y Buffer Pipes pH 7.2 (50 mM).

\section{Técnica}

Utilizar plasma libre de hemólisis de muestras de animales en ayunas. En tubos de ensayo limpios añadir:

\begin{tabular}{cccc}
\hline & BLANCO & ESTANDAR & MUESTRA \\
\hline MUESTRA (ul) & ---- & ---- & $10 \mathrm{ul}$. \\
ESTANDAR $(\mathrm{ul})$ & ----- & $10 \mathrm{ul}$. & ---- \\
REACTIVO $(\mathrm{ml})$ & $1 \mathrm{ml}$. & $1 \mathrm{ml}$. & $1 \mathrm{ml}$. \\
\hline
\end{tabular}

Mezclar e incubar por $5 \mathrm{~min}$. a $37^{\circ} \mathrm{C}$ (Baño María), Leer las absorbancias a $520 \mathrm{~nm}$. Llevando a cero el espectrofotómetro con el reactivo de trabajo (blanco). El color resultante es estable por al menos $30 \mathrm{~min}$.

\section{Cálculos}

\section{F.C-}

TRIGLICÉRIDOS $(\mathrm{mg} / \mathrm{dl})=$ Factor $\quad \mathrm{x}$ Absorbancia de Muestra

\section{Determinación de hdl-colesterol}

\section{a) Método}

Reactivo precipitante (Wiener)

\section{b) Fundamento del Método}

Las lipoproteínas de alta densidad (HDL) se separan precipitando selectivamente las lipoproteínas de baja y muy baja densidad (LDL y VLDL) mediante el agregado de ácido fosfotúngstico en presencia de iones magnesio.

Las HDL quedan en el sobrenadante separado por centrifugación, donde se realiza la determinación del colesterol ligado a las mismas, empleando el sistema enzimático Colesterol oxidasa/Peroxidasa con colorimetría según Trinder (Fenol/4-Aminofenazona).

\section{c) Reactivos}

Reactivo precipitante: solución de ácido fosfotúngstico $0.44 \mathrm{mmol} / 1$ y cloruro de magnesio $20 \mathrm{mmol} / 1$.

\section{d) Procedimiento}

En un tubo de Kahn medir 200ul de muestra, y agregar $500 u l$ de Reactivo precipitante.

Homogenizar agitando (sin invertir) durante 20 segundos y dejar 10 minutos en reposo a temperatura ambiente. Centrifugar 10 minutos a $4000 \mathrm{rpm}$. o 2 minutos a 12000 rpm. Usar el sobrenadante límpido como muestra.

En tres tubos marcados B, S y D, colocar:

\begin{tabular}{cccc}
\hline & B & S & D \\
\hline Sobrenadante & - & - & $200 \mathrm{ul}$ \\
$\begin{array}{c}\text { Standard } \\
\text { Rvo. de Trabajo } \\
\text { Colestat } \\
\text { enzimatico }\end{array}$ & - & $20 \mathrm{ul}$ & - \\
\hline
\end{tabular}

Mezclar e incubar por 15 minutos a $37^{\circ} \mathrm{C}$. Retirar del baño y enfriar. Leer a $505 \mathrm{~nm}$ en espectrofotómetro o en colorímetro con filtro verde (490-530nm), llevando a cero con el Blanco.

\section{Cálculo de los resultados}

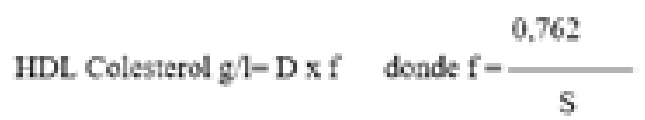

\section{Determinacion de LDL-Colesterol}

LDL-C $=\quad$ COLESTEROL
TRIGLICERIDOS $/ 5$

\section{Análisis histológico}

Se empleara el método de Harris

\section{a) Diagnóstico Histológico:}

El Diagnóstico patológico de las muestras obtenidas fueron sometidas a evaluación por el Laboratorio de Anatomía Patológica del Hospital Nacional CASE ESSALUD. Esta evaluación histopatológica se realizó en cada una de las láminas permanentes de los órganos en estudio (hígado, aorta), y para cada tratamiento utilizado.

\section{Análisis estadístico}

Los resultados obtenidos en los diferentes grupos de experimentación se utilizó el paquete estadístico SPSS 21 para Windows 8. Se Aplico la prueba de ANOVA y la postcomparación de Tukey $(\mathrm{p}<0.01)$. Análisis de Varianza: ANOVA o ANDEVA

\begin{tabular}{clllll}
\hline F de V & GL & SC & CM & Fc & Significancia \\
\hline Tratamientos & & & & & \\
Error & & & & & \\
Total & & & & & \\
\hline
\end{tabular}


En donde:

$\mathrm{F}$ de $\mathrm{V}=$ Fuentes de Variabilidad

Gl $\quad=$ Grados de Libertad

$\mathrm{SC} \quad=$ Suma de Cuadrados

$\mathrm{CM} \quad=$ Cuadrado Medio

$\mathrm{Fc} \quad=$ Prueba de $\mathrm{F}$.

Prueba de especificidad de Tukey: el nivel de significancia utilizado fue de $95 \%$

Coeficiente de variabilidad.

La significancia se evaluó en base a:

$\mathrm{p}<0.05$ diferencia significativa.

$\mathrm{p}<0.01$ diferencia altamente significativa.

$\mathrm{p} \geq 0.05$ diferencia no significativa.

\section{RESULTADOS}

Los resultados de nuestro trabajo los presentamos por medio de Tablas y Gráficos y la discusión de los mismos para evaluar el efecto hipocolesterolemiante de extracto acuoso de fruto de Solanum melongena "berenjena" en Rattus norvegicus variedad Wistar con hiperlipidemia experimental.

Tabla 1: Concentración de colesterol total $(\mathrm{mg} / \mathrm{dl})$ en Rattus norvegicus variedad wistar con hiperlipidemia experimental según tratamiento con extracto acuoso de Solanum melongena "berenjena"

\begin{tabular}{cccccccc}
\hline \multirow{2}{*}{$\begin{array}{c}\text { TRATAMIENTO/ } \\
\text { DIA }\end{array}$} & $\mathrm{N}$ & \multicolumn{6}{c}{ DIAS } \\
\cline { 3 - 8 } & BASAL & HIPER & 7 & 14 & 21 & 28 \\
\hline CONTROL & 6 & $75.29 \mathrm{a}$ & $112.44 \mathrm{a}$ & $106.55 \mathrm{~b}$ & $104.57 \mathrm{c}$ & $104.25 \mathrm{~d}$ & $105.63 \mathrm{c}$ \\
$\begin{array}{c}\text { S. melongena 0.5 g/ } \\
\text { kg/día }\end{array}$ & 6 & $75.57 \mathrm{a}$ & $109.64 \mathrm{a}$ & $102.91 \mathrm{a}$ & $95.26 \mathrm{~b}$ & $94.40 \mathrm{c}$ & $93.99 \mathrm{~b}$ \\
$\begin{array}{c}\text { S. melongena 1 g/ } \\
\text { kg/día }\end{array}$ & 6 & $76.02 \mathrm{a}$ & $113.64 \mathrm{a}$ & $106.92 \mathrm{~b}$ & $93.33 \mathrm{ab}$ & $81.27 \mathrm{~b}$ & $75.44 \mathrm{a}$ \\
$\begin{array}{c}\text { LOVASTATINA 3 } \\
\text { mg/kg/día }\end{array}$ & 6 & $78.05 \mathrm{a}$ & $112.73 \mathrm{a}$ & $102.63 \mathrm{a}$ & $91.20 \mathrm{a}$ & $76.62 \mathrm{a}$ & $73.32 \mathrm{a}$ \\
$\mathrm{F}$ & & 3.89 & 2.47 & 6.83 & 73.34 & 92.73 & 595.04 \\
P & $>0.05$ & $>0.05$ & $<0.01$ & $<0.01$ & $<0.01$ & $<0.01$ \\
\hline SIGNIFICANCIA & N.S. & N.S. & A.S. & A.S. & N.S. & N.S. \\
\hline
\end{tabular}

En la Tabla y Gráfico 1 se muestra la concentración de colesterol total, evidenciándose una disminución significativa en los tratamientos con extracto acuoso de fruto de Solanum melongena a una dosis de 0.5 y $1 \mathrm{~g} / \mathrm{kg} /$ día y lovastatina $3 \mathrm{mg} /$ $\mathrm{kg}$ en Rattus norvegicus variedad Wistar con hiperlipidemia experimental.

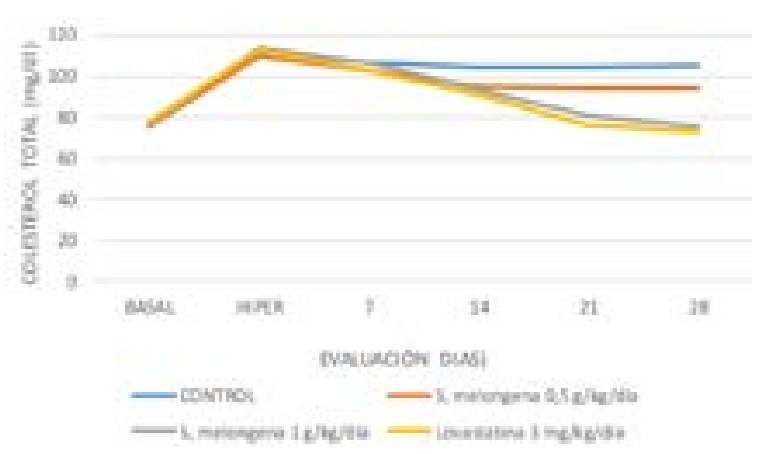

Gráfico 1: Concentración de colesterol total (mg/dl) en Rattus norvegicus variedad wistar con hiperlipidemia experimental según tratamiento con extracto acuoso de Solanum melongena "berenjena"
Tabla 2: Concentración de trigliceridos (mg/dl) en Rattus norvegicus variedad wistar con hiperlipidemia experimental según tratamiento con extracto acuoso de Solanum melongena "berenjena"

\begin{tabular}{cccccccc}
\hline TRATAMIENTO/DIA & $\mathrm{N}$ & BASAL & HIPER & 7 & 14 & 21 & 28 \\
\hline CONTROL & 6 & $75.07 \mathrm{a}$ & $98.13 \mathrm{a}$ & $95.80 \mathrm{c}$ & $95.16 \mathrm{~d}$ & $96.45 \mathrm{~d}$ & $95.34 \mathrm{~d}$ \\
S. melongena 0.5 g/kg/día & 6 & $75.58 \mathrm{a}$ & $97.75 \mathrm{a}$ & $94.93 \mathrm{bc}$ & $90.22 \mathrm{c}$ & $89.25 \mathrm{c}$ & $87.17 \mathrm{c}$ \\
& & & & & & & \\
S. melongena 1 g/kg/día & 6 & $74.59 \mathrm{a}$ & $98.58 \mathrm{a}$ & $92.19 \mathrm{a}$ & $83.68 \mathrm{~b}$ & $81.25 \mathrm{~b}$ & $77.36 \mathrm{~b}$ \\
$\begin{array}{c}\text { LOVASTATINA 3 mg/ } \\
\text { kg/día }\end{array}$ & 6 & $75.07 \mathrm{a}$ & $99.03 \mathrm{a}$ & $94.01 \mathrm{~b}$ & $81.32 \mathrm{a}$ & $75.66 \mathrm{a}$ & $74.93 \mathrm{a}$ \\
F & & 2.82 & 3.04 & 37.66 & 133.20 & 800.73 & 111.45 \\
P & & $>0.05$ & $>0.05$ & $<0.01$ & $<0.01$ & $<0.01$ & $<0.01$ \\
SIGNIFICANCIA & N.S. & N.S. & A.S. & A.S. & A.S. & A.S. \\
\hline
\end{tabular}

En la Tabla y Gráfico 2 se muestra la evolución de la concentración de triglicéridos, evidenciándose una disminución significativa en los tratamientos con extracto acuoso de Solanum melongena a una dosis de 0.5 y $1 \mathrm{~g} / \mathrm{kg} / \mathrm{día}$ y lovastatina $3 \mathrm{mg} / \mathrm{kg}$ en Rattus norvegicus variedad Wistar con hiperlipidemia experimental.

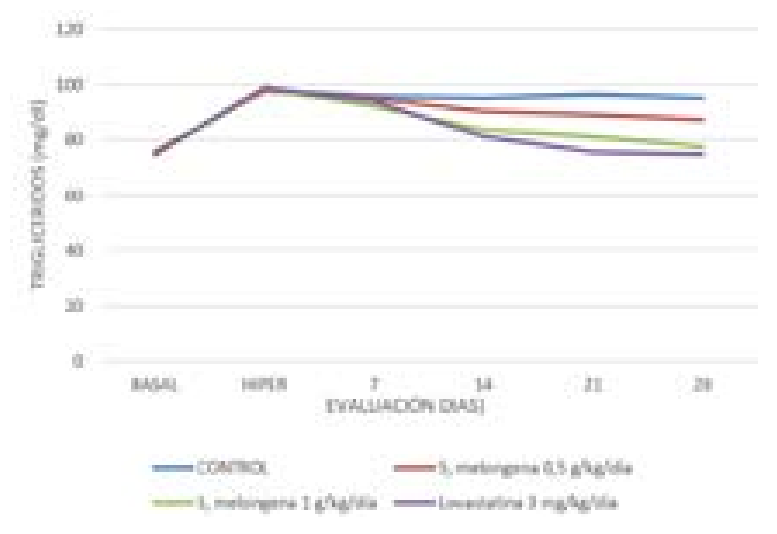

Gráfico 2: Concentración de trigliceridos (mg/dl) en Rattus norvegicus variedad wistar con hiperlipidemia experimental según tratamiento con extracto acuoso de Solanum melongena "berenjena"

Tabla 3: Concentración de LDL-Colesterol(mg/dl) en Rattus norvegicus variedad wistar con hiperlipidemia experimental según tratamiento con extracto acuoso de Solanum melongena "berenjena"

\begin{tabular}{cccccccc}
\hline TRATAMIENTO/DIA & $\mathrm{N}$ & BASAL & HIPER & 7 & 14 & 21 & 28 \\
\hline CONTROL & 6 & $11.33 \mathrm{a}$ & $37.47 \mathrm{a}$ & $38.70 \mathrm{c}$ & $38.73 \mathrm{~d}$ & $37.25 \mathrm{~d}$ & $36.92 \mathrm{~d}$ \\
& & & & & & & \\
S. melongena $0.5 \mathrm{~g} / \mathrm{kg} / \mathrm{dí}$ & 6 & $11.26 \mathrm{a}$ & $38.59 \mathrm{a}$ & $36.93 \mathrm{~b}$ & $31.99 \mathrm{c}$ & $30.52 \mathrm{c}$ & $29.81 \mathrm{c}$ \\
& & & & & & & \\
$\begin{array}{c}\text { S. melongena } 1 \mathrm{~g} / \mathrm{kg} / \mathrm{día} \\
\text { (6) }\end{array}$ & $11.33 \mathrm{a}$ & $38.22 \mathrm{a}$ & $35.68 \mathrm{~b}$ & $29.29 \mathrm{~b}$ & $25.15 \mathrm{~b}$ & $23.82 \mathrm{~b}$ \\
$\begin{array}{c}\text { LOVASTATINA 3 mg/ } \\
\mathrm{kg} / \text { día }\end{array}$ & 6 & $11.42 \mathrm{a}$ & $37.13 \mathrm{a}$ & $34.07 \mathrm{a}$ & $23.99 \mathrm{a}$ & $20.50 \mathrm{a}$ & $15.96 \mathrm{a}$ \\
F & & 1.46 & 3.42 & 24.03 & 229.44 & 293.81 & 239.14 \\
P & & $>0.05$ & $>0.05$ & $<0.01$ & $<0.01$ & $<0.01$ & $<0.01$ \\
SIGNIFICANCIA & N.S. & N.S. & A.S. & A.S. & A.S. & A.S. \\
\hline & & & & & &
\end{tabular}

En la Tabla y Gráfico 3 se muestra la evolución de la concentración de LDL-C, evidenciándose una disminución significativa en los tratamientos con extracto acuoso de Solanum melongena a una dosis de $0.5 \mathrm{~g} 1 \mathrm{~g} / \mathrm{kg} / \mathrm{día}$ y lovastatina $3 \mathrm{mg} / \mathrm{kg}$ en Rattus norvegicus variedad Wistar con hiperlipidemia experimental. 


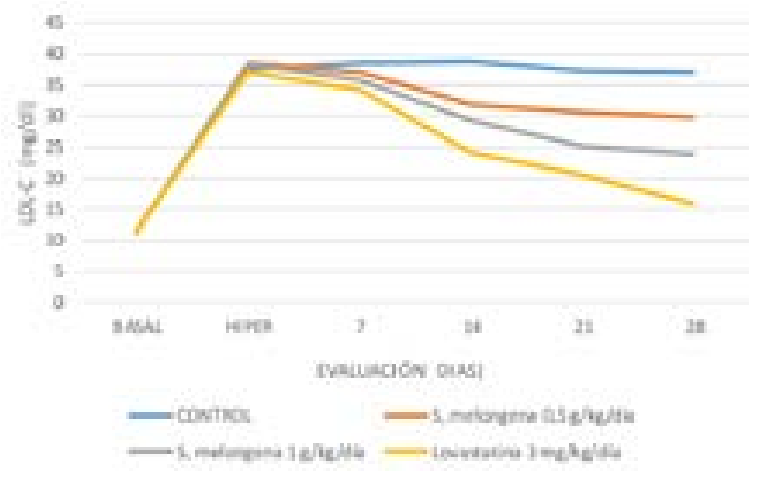

Gráfico 3: Concentración de Idl-colesterol(mg/dl) en Rattus norvegicus variedad wistar con hiperlipidemia experimental según tratamiento con extracto acuoso de Solanum melongena "berenjena"

Tabla 4: Concentración de hdl-colesterol $(\mathrm{mg} / \mathrm{dl})$ en Rattus norvegicus variedad wistar con hiperlipidemia experimental según tratamiento con extracto acuoso de Solanum melongena "berenjena"

\begin{tabular}{cccccccc}
\hline TRATAMIENTO/DIA & $\mathrm{N}$ & BASAL & HIPER & 7 & 14 & 21 & 28 \\
\hline CONTROL & 6 & $53.13 \mathrm{a}$ & $41.92 \mathrm{a}$ & $42.32 \mathrm{a}$ & $42.49 \mathrm{a}$ & $41.59 \mathrm{a}$ & $40.88 \mathrm{a}$ \\
S. melongena 0.5 g/kg/día & 6 & $52.52 \mathrm{a}$ & $42.58 \mathrm{a}$ & $43.63 \mathrm{ab}$ & $45.79 \mathrm{~b}$ & $45.00 \mathrm{~b}$ & $46.65 \mathrm{~b}$ \\
& & & & & & & \\
$\begin{array}{c}\text { S. melongena 1 g/kg/día } \\
\text { ONASTATINA 3 mg/ }\end{array}$ & 6 & $53.36 \mathrm{a}$ & $43.25 \mathrm{a}$ & $43.68 \mathrm{ab}$ & $48.01 \mathrm{c}$ & $49.15 \mathrm{c}$ & $49.55 \mathrm{c}$ \\
$\begin{array}{c}\text { kg/día } \\
\text { F }\end{array}$ & $52.86 \mathrm{a}$ & $42.52 \mathrm{a}$ & $44.94 \mathrm{~b}$ & $50.07 \mathrm{~d}$ & $50.86 \mathrm{~d}$ & $54.16 \mathrm{~d}$ \\
P & & 1.35 & 2.68 & 3.43 & 110.48 & 129.12 & 152.48 \\
SIGNIFICANCIA & & $>0.05$ & $>0.05$ & $<0.05$ & $<0.01$ & $<0.01$ & $<0.01$ \\
& N.S. & N.S. & S. & A.S. & A.S. & A.S. \\
\hline
\end{tabular}

En la Tabla y Gráfico 4 se muestra la evolución de la concentración de HDL-C, evidenciándose un aumento significativo en los tratamientos con extracto acuoso de Solanum melongena a una dosis de $0.5,1 \mathrm{~g} / \mathrm{kg} / \mathrm{día}$ y lovastatina $3 \mathrm{mg} / \mathrm{kg}$ en Rattus norvegicus variedad Wistar con hiperlipidemia experimental.

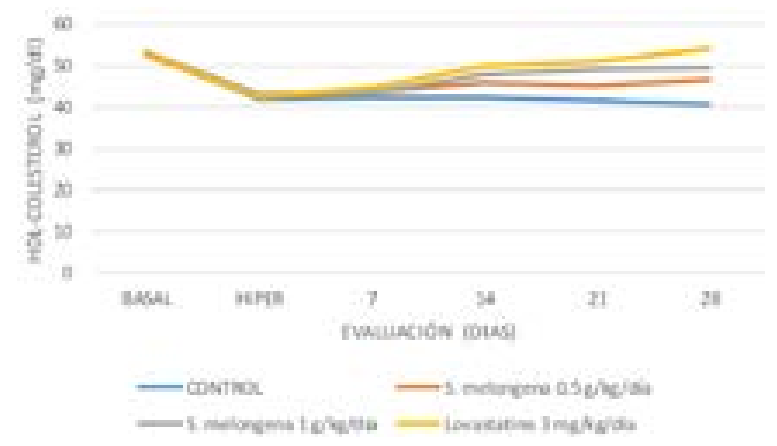

Gráfico 4: Concentración de hdl-colesterol(mg/dl) en Rattus norvegicus variedad wistar con hiperlipidemia experimental según tratamiento con extracto acuoso de Solanum melongena "berenjena"

\section{Análisis histológico}

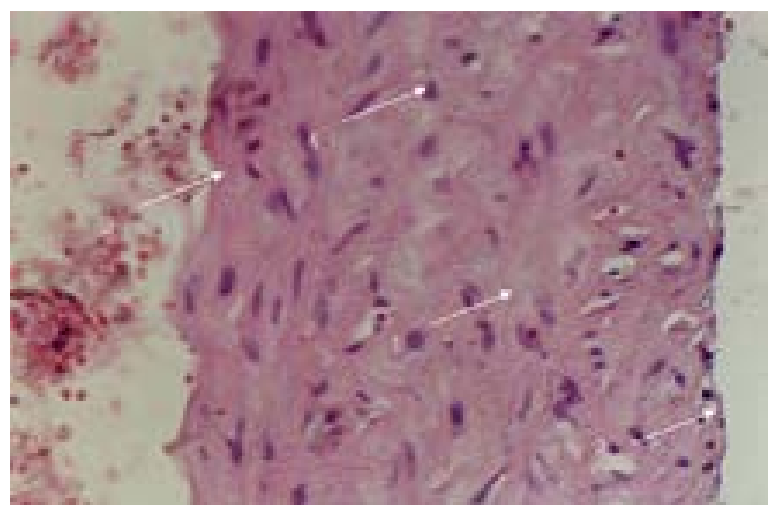

Fig. 1: Estructura de aorta de tratamiento control en Rattus norvegicus variedad wistar con hiperlipidemia experimental hematoxilina eosina $100 \mathrm{x}$

En esta microfotografía observamos la citoarquitectura normal de aorta corte longitudinal de corazón de ratas del grupo control

1: Membranas elásticas de la túnica media

2: Endotelio

3: Adventicia

4: Fibroblastos

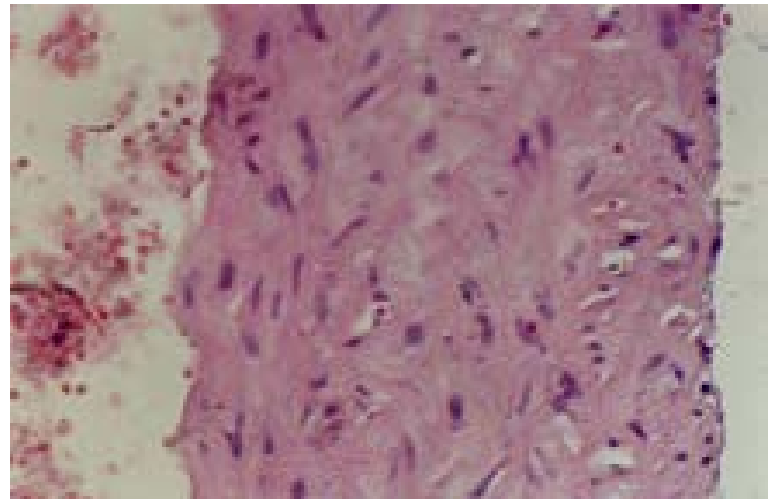

Fig. 2: Estructura de aorta de tratamiento de $1 \mathrm{~g} / \mathrm{kg} / \mathrm{dia}$ de extracto acuoso de Solanum melongena "berenjena" en Rattus norvegicus variedad wistar con hiperlipidemia experimental hematoxilina eosina $400 \mathrm{X}$

En esta microfotografía observamos la citoarquitectura de aorta corte longitudinal de corazón de ratas del grupo con tratamiento de $1 \mathrm{~g} / \mathrm{kg} /$ día de extracto acuoso de Solanum melongena.

1: Membranas elásticas de la túnica media

2: Endotelio

3: Adventicia 


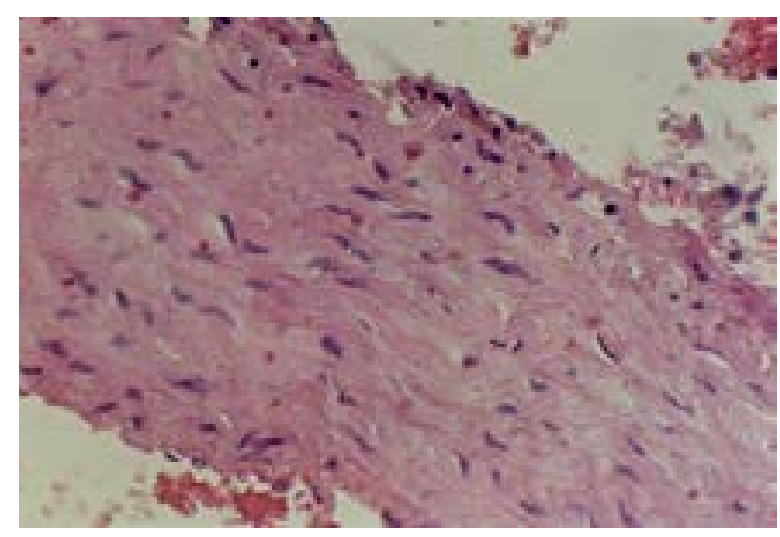

Fig. 3: Estructura de aorta de tratamiento de $3 \mathrm{mg} / \mathrm{kg} / \mathrm{dia}$ de lovastatina en Rattus norvegicus variedad wistar con hiperlipidemia experimental hematoxilina eosina $400 \mathrm{x}$

En esta microfotografía observamos la citoarquitectura de aorta corte longitudinal de corazón de ratas del grupo con tratamiento de $3 \mathrm{mg} / \mathrm{kg} /$ día de lovastatina.

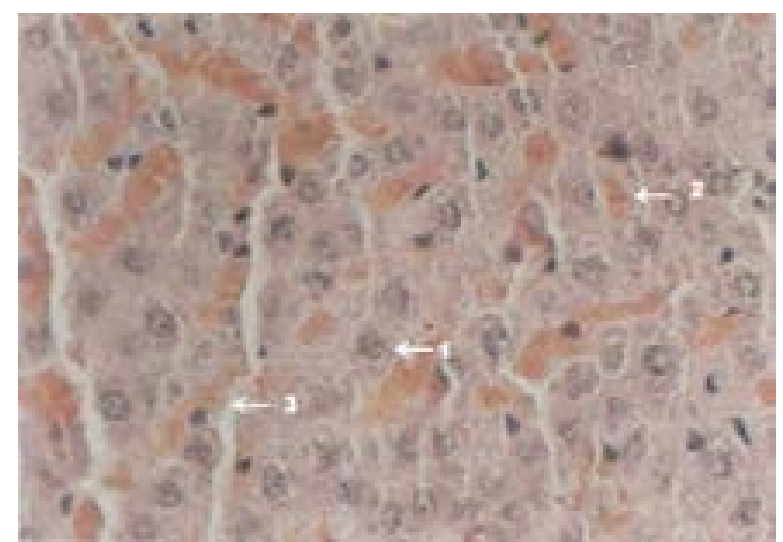

Fig. 4: Estructura de higado de tratamiento control en Rattus norvegicus variedad wistar con hiperlipidemia experimental hematoxilina eosina $400 \mathrm{X}$

En esta microfotografia (400X) de hígado de Rattus norvegicus variedad Wistar, observamos una citoarquitectura normal de los hepatocitos, es decir que se distribueyen en hileras, además los hepatocitos (1) se ven completamente definidas y con pared regular y conservada. La presencia de hematíes (2) en las venas es mínima y el parénquima hepático se nota uniforme y es posible observar los lobulillos hepáticos con los espacios sinusoidales (3) permeables.

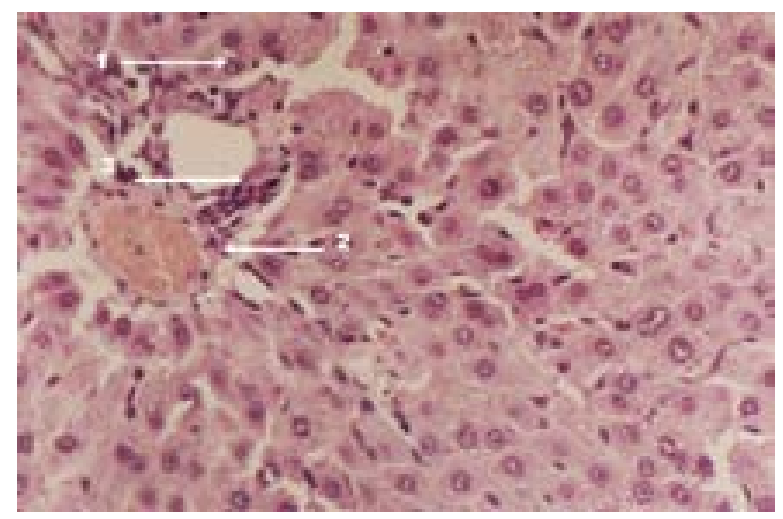

Fig.5: Estructura de higado de tratamiento de $1 \mathrm{~g} / \mathrm{kg} / \mathrm{dia}$ de extracto acuoso de Solanum melongena "berenjena" en Rattus norvegicus variedad wistar con hiperlipidemia experimental hematoxilina eosina $400 \mathrm{X}$
En esta microfotografía observamos la citoarquitectura normal de hígado de ratas del grupo control. Se puede observar:

1: Hepatocitos

2: Vasos sanguíneos

3: Porta hepatica

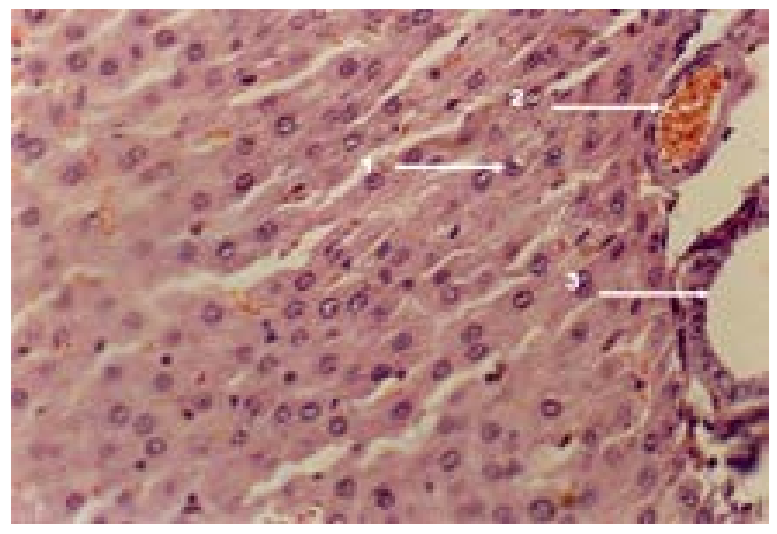

Fig. 6: Estructura de higado de tratamiento de $3 \mathrm{mg} / \mathrm{kg} / \mathrm{dia}$ de lovastatina en Rattus norvegicus variedad wistar con hiperlipidemia experimental hematoxilina eosina $400 \mathrm{X}$

En esta microfotografía observamos hígado de ratas del grupo con lovastatina en dosis de $3 \mathrm{mg} / \mathrm{kg} /$ día. Se puede observar estructura normal:

1: Hepatocitos

2: Vasos sanguíneos

3: Porta hepatica

\section{DISCUSIÓN}

Nuestro trabajo de investigación confirma en primer lugar que la dieta aterogénica formulada fue efectiva en producir índices aterogénicos elevados en Rattus norvegicus variedad Wistar.

El consumo elevado de grasas y colesterol, variable independiente en nuestro trabajo, es sin duda la causa primaria de la aterogénesis que incrementa in vivo la producción de radicales libres que producen la peroxidación lipídica y modificación oxidativa de LDL-C, el mayor portador de colesterol sérico, lo que hace que sean fácilmente fagocitadas por los macrofagos (MAC). Efectivamente la LDL-C es pobremente metabolizada por MAC, pero cuando LDL-C está oxidada lo es con facilidad.

Los resultados muestran que la administración de 0.5 y 1 $\mathrm{g} / \mathrm{kg} /$ día de extracto acuoso de fruto de Solanum melongena "berenjena" produce disminución significativa $(p<0.01)$ en los niveles de colesterol total y triglicéridos en ratas con hiperlipidemia experimental. Estos resultados corroboran con lo reportado por Duke (1992), Botelho y col (2004), Guimarae y col (2000).

El lovastatina es efectivo para reducir la concentración de colesterol total y triglicéridos, debido a que inhibe competitivamente la HMG CoA reductasa. La inhibición de la HMG CoA reductasa previene la conversión de la HMG CoA a mevalonato, un paso limitante de la velocidad en la biosíntesis del colesterol. La inhibición de la síntesis del colesterol en el hígado conduce a una disminución de los receptores LDL y un aumento del catabolismo del colesterol LDL. También puede haber una reducción en la producción de LDL como resultado de la inhibición de la 
síntesis hepática de VLDL, precursor del LDL. es probable que actúe inhibiendo la secreción hepática de VLDL, inhibe la lipólisis de triglicéridos almacenados en el tejido adiposo y disminuye la captación de ácidos grasos por el hígado. Lo que permite establecer la disminución significativa de triglicéridos, colesterol, LDL-C, e indices aterogénicos (Goodman G. 2002).

El extracto acuoso de Solanum melongena "berenjena" tiene acción hipocolesterolémica e hipolipidémica, porque dentro de sus constituyentes fitoquímicos presenta elementos que disminuyen los niveles de colesterol total y triglicéridos. Davies y Stewart (1990) reportan la presencia de acido esteárico, ácido clorogénico y ácido linoleico. Estos ácidos grasos insaturados ocupan un área mayor que los saturados, en razón de su configuración espacial. De este modo se incorporarían menos moléculas lipídicas a las apoproteínas de las LDL $-\mathrm{C}$, con lo que disminuirían el riesgo de hiperlipemias y aterosclerosis.

Werbach (1993) reporta la presencia de fitosterol, licopeno. Son esteroles vegetales con efecto hipolipemiante, debido a su similitud estructural con el colesterol, compiten con éste por la solubilización en micelas; de este modo, inhiben la absorción tanto del colesterol de la dieta como el endógeno.

Pizzorno (1985) reporta la presencia de magnesio, cromo, cobre, calcio, quienes contribuirían en reducir los niveles de glucosa y colesterol, lo que resulta muy beneficioso en la obesidad, y podría ayudar a reducir masa grasa. Además, se han demostrado las acciones antioxidante, antiglicación,antiinflamatoria y detoxificante.

Duke (1992) reporta la presencia de niacina (ácido nicotínico) El ácido nicotínico disminuye los niveles plasmáticos de colesterol (total, LDL y VLDL-colesterol), triglicéridos, lipoproteína A (partícula LDL modificada) y apolipoproteína $\mathrm{B}$, principal proteína constitutiva de las partículas LDL. De igual manera aumenta los niveles de HDL-colesterol y de apolipoproteína A1, principal componente proteínico de esta lipoproteína.

Duke (1992) reporta pectina y fibra que reduce lipoproteínas de baja densidad, sin alteraciones de las lipoproteínas de alta densidad o de los niveles de triglicéridos. El mecanismo probable es la reducción de la reserva hepática de colesterol, la modificación de las enzimas que regulan la homeostasis del colesterol, la sobreregulación del receptor hepático de las lipoproteínas de baja densidad y el aumento de la extracción de las lipoproteínas de baja densidad del plasma.

Huang (2003) reporta la presencia de trigonelina debido a que interaccionan con las sales biliares disminuye los niveles de colesterol y riesgo de aterosclerosis.

Además es necesario señalar que extracto acuoso de Solanum melongena "berenjena" dentro de su composición fitoquímica presenta componentes antiateroscleróticos. Pizzorno (1985) reporta ácido ascórbico. Davies y Stewart (1990) reporta ácido linoleíco. Duke (1992) reporta niacina.

Por lo anteriormente señalado, se puede establecer, probablemente, la acción hipolipemiante del extracto acuoso de fruto de Solanum melongena "berenjena". Dichos componentes fitoquímicos juegan un rol importante en el metabolismo lipídico, dichos resultados se corroboran por lo descrito por Urrea y col (1995) quienes reportan disminución de los niveles de colesterol e índices aterogénicos. Goncalves y col (2006) quienes evaluaron la acción hipocolesteromiante de extracto acuoso de Solanum melongena "berenjena" en el perfil lipídico en ratas con hiperlipidemia. Reportando que el extracto acuoso de Solanum melongena "berenjena" disminuye los niveles de colesterol Botelho y col (2004), Guimaraes y col (2000).

\section{REFERENCIAS BIBLIOGRÁFICAS}

1. BALDARRAGO, S.G. CABRERA V.P. (1993), Sistematización de vademecum Fitoterapeútico. Tomo IV Región Arequipa.

2. BOTELHO FV, ENÉAS LR, CESAR GC, BIZZOTTO CS, TAVARES E, OLIVEIRA FA, GLORIA MB, SILVESTRE MP, ARANTES RM, ALVAREZ-LEITE JI. Effects of eggplant (Solanum melongena) on the atherogenesis and oxidative stress in LDL receptor knock out mice (LDLR(-/-)). Food Chem Toxicol. 2004 Aug;42(8):1259-67.

3. DAVIES, S., y STEWART, A. 1990. Nutritional Medicine. Avon Books, New York. 509pp.

4. DUKE, JAMES A. 1992. Handbook of biologically active phytochemicals and their activities. Boca Raton, FL. CRC Press.

5. GANONG W.F. Fisiología Médica. 2011. Editorial El Manual Moderno S.A. de México.

6. GINZINGER D.G., WILSON J.E., REDENBACH D., LEWIS S., CLEE S.M., ASHBOURNE J.D., ROGERS, Q.R., HAYDEN M.R., AND McMANUS B.M. 1997. Diet-induced atherosclerosis in the domestic cat. Laboratory Investigation. 77(5):409-419.

7. GONÇALVES, M. C.; DINIZ, M. F. F. M.; DANTAS, A. H.G.; BORBA, J. D. C. 2006. Modesto efeito hipolipemiante do extrato seco de berinjela (Solanum melongena L.) em mulheres com dislipidemias, sob controle nutricional. Brazilian Journal of Pharmacognosy 16(Supl.): 656-663,

8. GOODMAN G., GOODMAN L., RALL T. MURAD F. 2002. Las Bases Farmacológicas de la Terapeútica. Ed. Panamericana. Buenos Aires.

9. GUIMARÃES PR, GALVÃO AM, BATISTA CM, AZEVEDO GS, OLIVEIRA RD, LAMOUNIER RP, FREIRE N, BARROS AM, SAKURAI E, OLIVEIRA JP, VIEIRA EC, ALVAREZ-LEITE JI. Eggplant (Solanum melongena) infusion has a modest and transitory effect on hypercholesterolemic subjects. Braz J Med Biol Res. 2000 Sep;33(9):1027-36.

10. GUYTON A.C. AND HALL J.E. 2012. Tratado de Fisiología Médica. McGraw-Hill, Interamericana. México

11. HUANG, K. C. 2003. The Pharmacology of Chinese Herbs. CRC Press, Boca Raton, FL 388 pp.

12. MACHACA S. D. 1998. Efecto del extracto crudo de Portulaca oleracea L. "verdolaga"en niveles séricos de colesterol total y triglicéridos en pacientes hipercolesterolémicos y normolcolesterolémicos. Tesis para Biólogo. UNSA.

13. MURRAY, R. MAYES, P. GRANNER, D. RODWELL, V. 2011. Bioquímica de Harper. Editorial El Manual Moderno. México.

14. PIZZORNO, J.E. y MURRAY, M.T. 1985. A Textbook of Natural Medicine. John Bastyr College Publications, 
Seattle, Washington (Looseleaf).

15. PONTE PI, MENDES I, QUARESMA M, AGUIAR MN, LEMOS JP, FERREIRA LM, SOARES MA, ALFAIA CM, PRATES JA, FONTES CM Cholesterol levels and sensory characteristics of meat from broilers consuming moderate to high levels of alfalfa. : Poult Sci. 2004 May;83(5):810-4

16. RING, M.A. 1989. Ethnopharmacologic Analysis of Medicinal Plants Used by Laotian Hmong Refugees in Minnesota. Journal of Ethnopharmacology, 26: 65-91, 1989.

17. ROBBINS. Patología funcional y estructural, $6^{\mathrm{a}}$ ed., Ed. Interamericana, 2000.

18. RUBIN R. y FARBER R. 2010. Patología Estructural y Funcional. . Editorial Panamericana.

19. STORY JA, LEPAGE SL, PETRO MS, WEST LG, CASSIDY MM, LIGHTFOOT FG, VAHOUNY GV.Interactions of alfalfa plant and sprout saponins with cholesterol in vitro and in cholesterol-fed rats. Am J Clin Nutr. 1984 Jun;39(6):917-29.

20. STRYER, L. 2008. Fundamentos de Bioquímica. Editorial Reverte Barcelona España

21. URREA, D. POLO, A. SANABRIA, A. (1995). Actividad hipolipidica del exctracto acuoso del fruto de Solanum melongena. Rev. Colombiana de Ciencias Químico Farmaceúticas Nro. 23 13-17

22. WERBACH, M. 1993. Healing with Food. Harper Collins, New York, 443 pp.

23. WICK G, KLEINDIENSTR, SCHETT G,AMBERGER A, XU Q. 1995. Role of heat shock protein $65 / 60$ in the pathogenesis of atherosclerosis. Int-Arch-AllergyImmunol. May-Jun; 107(1-3): 130-1. 